\title{
Dynamics of coagulation factor XIII activity after video-assisted thoracoscopic lobectomy for non-small cell lung cancer
}

\author{
Suguru Shirai, Yoshikane Yamauchi, Fumi Yokote, Takashi Sakai, Yuichi Saito, Yukinori Sakao, \\ Masafumi Kawamura \\ Department of Surgery, Teikyo University School of Medicine, Tokyo, Japan \\ Contributions: (I) Conception and design: S Shirai, Y Yamauchi; (II) Administrative support: Y Yamauchi, Y Sakao, M Kawamura; (III) Provision of \\ study materials or patients: S Shirai, Y Yamauchi, Y Saito, Y Sakao; (IV) Collection and assembly of data: S Shirai, F Yokote, T Sakai, Y Sakao; (V) \\ Data analysis and interpretation: S Shirai, Y Yamauchi, Y Sakao, MK; (VI) Manuscript writing: All authors; (VII) Final approval of manuscript: All \\ authors. \\ Correspondence to: Yoshikane Yamauchi, MD, PhD. Department of Surgery, Teikyo University School of Medicine, Kaga 2-11-1, Itabashi, Tokyo 173- \\ 8605, Japan. Email: yoshikaney@med.teikyo-u.ac.jp.
}

\begin{abstract}
Background: The present study was performed to investigate the perioperative dynamics of coagulation factor XIII (FXIII) in patients with non-small cell lung cancer undergoing video-assisted thoracoscopic surgery (VATS) lobectomy compared with open lobectomy.

Methods: Perioperative coagulation factors including FXIII were analyzed in 30 patients who underwent VATS lobectomy and 10 patients who underwent open lobectomy at Teikyo University Hospital from December 2017 to April 2019.

Results: Patients in the VATS lobectomy group showed higher FXIII activity on postoperative day (POD) 5 than patients in the open lobectomy group $(\mathrm{P}=0.028)$. The FXIII activity was significantly lower on POD3, POD5, and POD7 than that in the preoperative period and on POD1, even in patients who had undergone VATS lobectomy $(\mathrm{P}<0.001)$. No factors were found to affect the maintenance of FXIII in the VATS lobectomy group.

Conclusion: The postoperative decrease of FXIII activity differed between patients who underwent VATS lobectomy and those who underwent open lobectomy. Based on the characteristics of FXIII, the FXIII activity may be a good marker of the invasiveness of VATS lobectomy versus open lobectomy.
\end{abstract}

Keywords: Coagulation factor XIII (coagulation FXIII); non-small cell lung cancer (NSCLC); video-assisted thoracic surgery (VATS); surgical invasiveness

Submitted Sep 25, 2019. Accepted for publication Nov 12, 2019.

doi: $10.21037 /$ jtd.2019.12.08

View this article at: http://dx.doi.org/10.21037/jtd.2019.12.08

\section{Introduction}

Video-assisted thoracoscopic surgery (VATS) lobectomy is now frequently used as the curative surgical approach in patients with non-small cell lung cancer (NSCLC) (1). General thoracic surgeons generally tend to consider that VATS lobectomy must be less invasive than open lobectomy. Many published papers have suggested that VATS lobectomy is an adequate oncological operation, is safe, is better tolerated than open lobectomy, and facilitates the delivery of adjuvant chemotherapy (2-5). Moreover, several multicenter case-control studies have shown that the potential benefits of VATS include less pain, chest drainage, length of hospitalization, and complications (6-9). However, these benefits are not as statistically robust in prospective randomized trials. Moreover, inflammation markers, such as C-reactive protein or erythrocyte sedimentation rate, can be easily affected by other physical factors, therefore they are not always suitable for the evaluation of surgical invasiveness. 
Blood coagulation factor XIII (FXIII) is not only a key factor that functions at the end of the coagulation cascade, but it also plays an important role in wound healing, making cross-linking fibrin monomers into stable polymers and counteracting fibrinolytic degradation (10). Administration of exogenous FXIII concentrate reportedly aids wound healing (11-14) and intestinal anastomosis leakage after gastrointestinal surgery $(15,16)$. Moreover, Inoue et al. (17) reported that FXIII was not only an important factor for prolonged air leakage after pulmonary resection but also that exogenous FXIII helped to treat the air leak.

To assess the difference in surgical invasiveness between VATS lobectomy and open lobectomy, we focused on the area of surgical trauma on the chest wall because surgical invasiveness is presumed to be correlated with this area of surgical trauma. From this viewpoint, we considered that the invasiveness could be directly measured by the factors involved in the wound healing system and that FXIII would be a good marker for the evaluation of invasiveness, because it is an important factor of wound healing.

The present study was performed to investigate the perioperative dynamics of FXIII in patients with NSCLC undergoing VATS lobectomy compared with open lobectomy and to investigate how these dynamics correlate with those of other perioperative parameters in patients undergoing VATS lobectomy.

\section{Methods}

\section{Patients}

This study was approved by the ethics review board of Teikyo University (approval number: 17-035). Thirty patients who underwent VATS lobectomy at the Department of Surgery, Teikyo University Hospital from December 2017 to April 2019 and 10 patients who underwent open lobectomy at the same hospital during the same period were analyzed. All 40 patients were consecutive cases and were preoperatively diagnosed with NSCLC without nodal metastasis by the institutional tumor board. PET scan was basically performed in all cases, except the patients had severe diabetes. In our institution, all patients diagnosed with clinical N0 lung cancer are candidates for VATS lobectomy. However, when patients chose to undergo open lobectomy instead of VATS lobectomy after our explanation of the difference between open lobectomy and VATS lobectomy, we preoperatively plan to perform open lobectomy.
Patients who underwent intraoperative conversion from VATS to open lobectomy were excluded. Patients who received a perioperative transfusion were also excluded because coagulation factors may be strongly affected by transfusion.

The preoperative characteristics were the patient's age, sex, smoking history, body mass index, preoperative diagnosis and tumor position, various nutrition indexes, and presence of comorbidities. The intraoperative parameters were the surgical procedure, operation time, and blood loss. Intraoperative blood loss was calculated by weighing blood-soaked gauze and measuring the amount of blood in suction bottles. Postoperative complications that occurred within 28 days after surgery were monitored and classified according to the Clavien-Dindo classification of surgical complications (18). The index of prolonged air leakage (IPAL) (19) was also calculated from the patients' information.

\section{Perioperative management}

Perioperative antimicrobial prophylaxis was performed immediately before surgery and every 3 to $12 \mathrm{~h}$ during and after surgery (the intervals were determined according to the half-lives of the antimicrobials) until 2 days after surgery. No patients underwent postoperative replacement of FXIII, even if prolonged air leakage was found.

\section{Surgical techniques}

All patients underwent lobectomy with mediastinal lymph node dissection. VATS lobectomy was performed entirely through four small incisions that measured 30, 15, 7, and $7 \mathrm{~mm}$, respectively, without rib spreading. A 5-mm highdefinition camera (Olympus, Tokyo, Japan) was inserted with 30-degree angulation. The operation was performed entirely through thoracoscopic images. In contrast, open lobectomy involved the performance of lobectomy following thoracotomy with rib spreading. The length of the skin incision was about $120 \mathrm{~mm}$.

\section{Blood samples for analysis}

Blood samples for analysis were obtained within 1 week before surgery and on postoperative day (POD) 1, POD3, POD5, and POD7. We measured the FXIII activity, fibrinogen level, fibrinogen degradation products (FDP) level, and complete blood count. All laboratory tests were performed according to the manufacturer's protocol. FXIII activity was measured via 
the synthetic substrate method (20). All patients' data were prospectively collected.

\section{Statistical analysis}

Statistical analyses were performed using Prism 8 (GraphPad Software Inc., San Diego, CA, USA). The results were assessed using unpaired or paired twotailed Student's $t$ tests. A value of $\mathrm{P}<0.05$ was considered statistically significant. The optimal cutoff values for continuous prognostic indexes were determined with the method established by Budczies et al. (21), described at http://molpath.charite.de/cutoff/. This method fits Cox proportional hazard models to the dichotomized variable and the survival variable. The optimal cutoff is defined as the point with the most significant split.

\section{Results}

\section{Patients' perioperative characteristics}

The patients' baseline characteristics are shown in Table 1. Thirty patients who underwent VATS lobectomy and 10 patients who underwent open lobectomy were included in this study. There was no significant difference in age, sex, body mass index, Glasgow prognostic score, or existence of diabetes between the two groups, although the smoking history was heavier in the open lobectomy group and tumor size is larger in the open lobectomy group. Additionally, the IPAL did not differ between the two groups in our study.

Among the preoperative data, there was no significant difference in the operation time, duration of drainage, or prolonged air leakage between the two groups, although patients in the open lobectomy group lost $200 \mathrm{~mL}$ more blood than those in the VATS lobectomy group (235 vs. $31 \mathrm{~mL}$ in average, $\mathrm{P}<0.001)$. There was also no significant difference in the tumor location, pathological stage, or histology between the two groups. Among the preoperative laboratory data, there was no significant difference in the FXIII activity, prothrombin time, activate partial thromboplastin time, fibrinogen level, FDP level, or platelet count between the two groups.

\section{Dynamics of perioperative FXIII activity and other coagulation factors}

Figure 1 shows the following perioperative laboratory data in both the VATS lobectomy group and open lobectomy group: FXIII activity (Figure $1 A$ ), fibrinogen level (Figure 1B), platelet count (Figure 1C), and FDP level (Figure 1D).

The FXIII activity was significantly lower on POD3, POD5, and POD7 than during the preoperative period (control) in the VATS lobectomy group $(\mathrm{P}<0.001)$. The fibrinogen level was significantly higher on POD3, POD5, and POD7 than during the preoperative period in the VATS lobectomy group $(\mathrm{P}<0.001)$. The platelet count was significantly lower on POD1 and POD 3 than during the preoperative period in the VATS lobectomy group $(\mathrm{P}<0.001)$, but it recovered on POD5 and POD7. The FDP level was significantly higher on POD5 and POD7 than during the preoperative period in the VATS lobectomy group $(\mathrm{P}<0.001)$.

In the comparison between VATS lobectomy and open lobectomy patients in the VATS lobectomy group showed higher FXIII activity on POD5 than those in the open lobectomy group $(\mathrm{P}=0.028)$. However, no significant difference was found in the other parameters, including the fibrinogen level, platelet count, and FDP level.

We dichotomized the VATS lobectomy group into two subgroups to identify parameters that affect the maintenance of FXIII. Figure 2 shows the comparison of FXIII activity according to the drainage volume (Figure 2A), HbA1c level (Figure 2B), prolonged air leakage (Figure $2 C$ ), operation time (Figure $2 D$ ), tumor size (Figure 2E), and intraoperative blood loss (Figure $2 F$ ). However, no factor reached statistical significance at any time point.

\section{Discussion}

In this study, we assessed the dynamics of preoperative wound healing factors in patients who underwent VATS lobectomy versus open lobectomy. Our data showed that the FXIII activity on POD5 was higher in the VATS lobectomy group than open lobectomy group. However, the FXIII activity was significantly lower on POD3, POD5, and POD7 than during the preoperative period in the VATS lobectomy group. Therefore, the difference in FXIII activity on POD5 depended on the declining range of FXIII activity between the VATS lobectomy group and open lobectomy group. To the best of our knowledge, no other report has described the perioperative dynamics of FXIII in patients undergoing VATS lobectomy versus open lobectomy.

The role of FXIII in wound healing is supported by 
Table 1 Patient characteristics

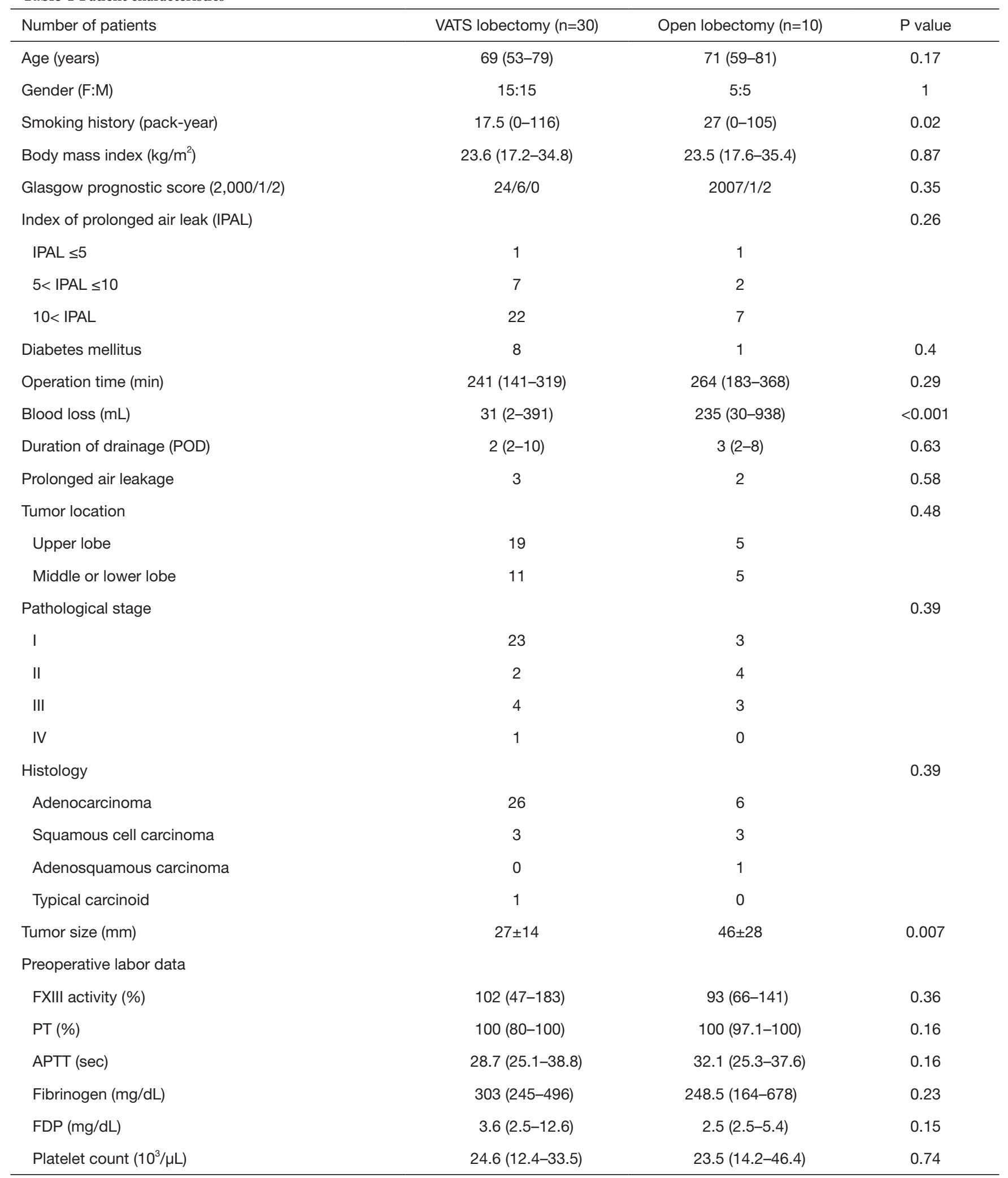

VATS, video-assisted thoracoscopic surgery; POD, postoperative day; FXIII, factor XIII; PT, prothrombin time; APTT, activated partial thromboplastin time; FDP, fibrinogen degradation products. 

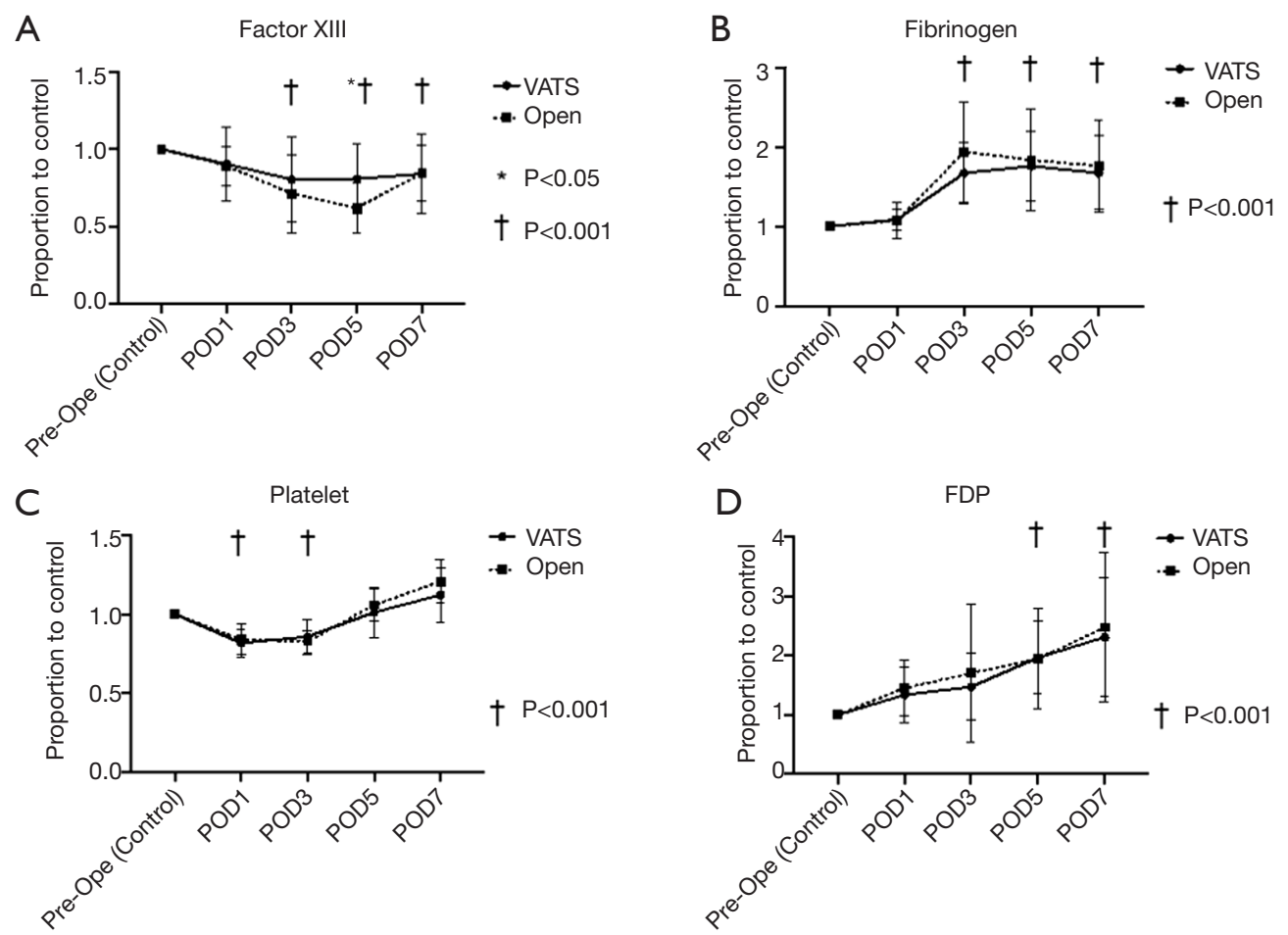

Figure 1 Dynamics of the following perioperative coagulation factors are shown: (A) FXIII, (B) fibrinogen, (C) FDP, and (D) platelet count. These data are described as the proportion to the preoperative data (control). ${ }^{\dagger}$, significant difference compared with preoperative control in the VATS lobectomy group; *, significant difference at the same time point between the VATS lobectomy and open lobectomy group. Higher FXIII activity on POD5 was found in the VATS lobectomy than open lobectomy group (P=0.028). The FXIII activity was significantly lower on POD3, POD5, and POD7 than during the preoperative period or POD1, even in the VATS lobectomy group $(\mathrm{P}<0.001)$. FXIII, factor XIII; FDP, fibrinogen degradation products; VATS, video-assisted thoracoscopic surgery; POD, postoperative day.

research in both animal studies and clinical cases. Schroeder and Kohler (22) reported that activated FXIII cross-links fibrin chains in a transglutaminase reaction, leading to a three-dimensional insoluble fibrin network that supports wound healing. Additionally, Higaki et al. (23) reported that the plasma FXIII level was lower in patients with active Crohn's disease than in patients in remission, indicating FXIII consumption during the healing of damaged colons. Moreover, D'Argenio et al. (24) evaluated patients with ulcerative colitis and found that FXIII was localized in the extracellular matrix of the damaged mucosa as revealed by immunohistochemistry, which is strong evidence of the participation of FXIII in the wound healing process.

How to supply FXIII to the healing tissue remains unresolved. Katona et al. (25) examined children with chronic bronchoalveolar inflammation and found that the plasma type FXIII level in bronchoalveolar lavage fluid was elevated, as was the cellular form of FXIII. From this point of view, the consumption of FXIII in our study may reflect the result of FXIII translocation in the healing tissue. Therefore, our findings suggest that FXIII activity may reflect the difference in surgical invasiveness between VATS lobectomy and open lobectomy.

FXIII also interacts with cells of the immune system (26). Interactions include activation of FXIII by human neutrophil elastase, down-regulation of activated FXIII within the clot by granulocyte proteases, and FXIIIinduced enhancement of monocyte proliferation and migration and inhibition of monocyte apoptosis (26). Monocytes and macrophages have been discussed as a source of FXIII-A in plasma, and in spite of some evidence for a non-classic secretion pathway of cellular FXIII from these cells $(26,27)$, the origin of plasma FXIII-A is not yet proven. An association between the FXIIIVal34Leu polymorphism and monocyte and neutrophil cell counts after lipopolysaccharide infusions in humans has been 

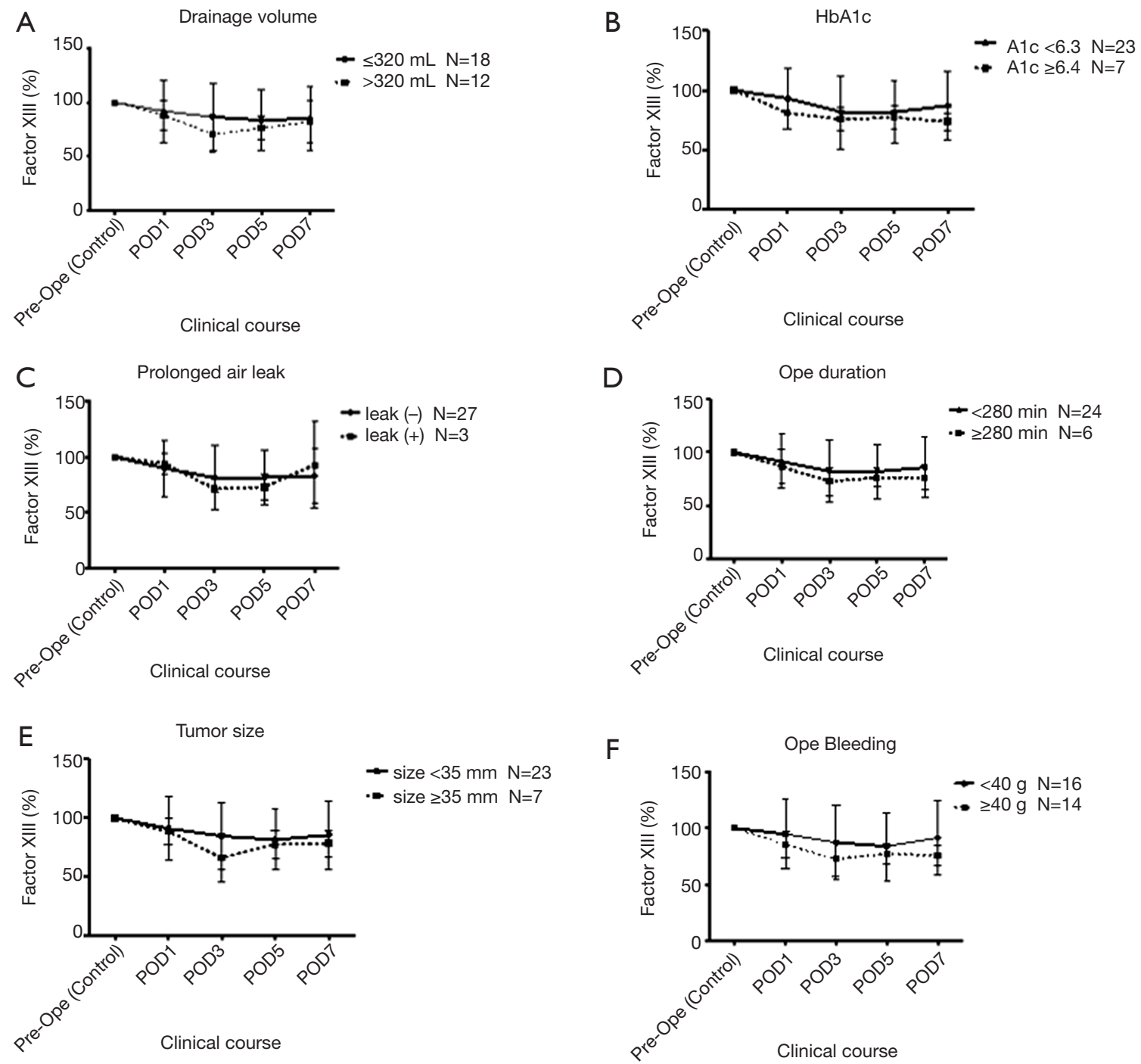

Figure 2 Dynamics of FXIII activity are shown in the VATS lobectomy group, with the groups dichotomized according to (A) drainage volume, (B) HbA1c level, (C) prolonged air leakage, (D) operation time, (E) tumor size, and (F) intraoperative blood loss. No factor reached a significant difference at any time point. FXIII, factor XIII; VATS, video-assisted thoracoscopic surgery.

suggested (28). VATS lobectomy for early-stage NSCLC has been found to be less immunosuppressive than open lobectomy as shown by less severely suppressed numbers of lymphocytes, total T cells, CD4 T cells, and NK cells in the early postoperative period $(29,30)$.

From these previous papers and our results, the maintenance of FXIII activity may be the result of the less invasive nature of VATS lobectomy. However, this may also result in the prevention of immunosuppression after surgery, which may contribute to better perioperative outcomes.

This study has several potential limitations. First, the number of enrolled patients was small. Additionally, the study was open-label and non-randomized; therefore, it is hard to know if this trend will be a true finding, because sampling bias may have occurred. In order to prove this trend statistically, randomized study with more patients will be required. Second, we described all coagulation factor data as proportions to the preoperative data; therefore, patients with poor coagulation factors tended to show drastic changes, even if the same amount of coagulation factors was consumed. However, the preoperative data of these patients showed wide variation, and proportion data are thus suitable for the comparison between the 
preoperative and postoperative periods.

In conclusion, we found that the FXIII activity differed between patients undergoing VATS lobectomy and those undergoing open lobectomy. Based on the characteristics of FXIII, the FXIII activity may be a good marker of surgical invasiveness in patients with NSCLC undergoing lobectomy.

\section{Acknowledgments}

We thank Angela Morben, DVM, ELS, from Edanz Group (www.edanzediting.com/ac), for editing a draft of this manuscript. A research fund from the Teikyo University School of Medicine supported this study.

\section{Footnote}

Conflicts of Interest: The authors have no conflicts of interest to declare.

Ethical Statement: The authors are accountable for all aspects of the work in ensuring that questions related to the accuracy or integrity of any part of the work are appropriately investigated and resolved. This study was approved by the ethics review board of Teikyo University (approval number: 17-035).

\section{References}

1. Howington JA, Blum MG, Chang AC, et al. Treatment of Stage I and II Non-small Cell Lung Cancer. Chest 2013;143:e278S-e313S.

2. Shigemura N, Akashi A, Funaki S, et al. Long-term outcomes after a variety of video-assisted thoracoscopic lobectomy approaches for clinical stage IA lung cancer: a multi-institutional study. J Thorac Cardiovasc Surg 2006;132:507-12.

3. Walker WS, Codispoti M, Soon SY, et al. Long-term outcomes following VATS lobectomy for non-small cell bronchogenic carcinoma. Eur J Cardiothorac Surg 2003;23:397-402.

4. Petersen RP, Pham D, Burfeind WR, et al. Thoracoscopic lobectomy facilitates the delivery of chemotherapy after resection for lung cancer. Ann Thorac Surg 2007;83:1245-9.

5. Thomas P, Doddoli C, Yena S, et al. VATS is an adequate oncological operation for stage I non-small cell lung cancer. Eur J Cardiothorac Surg 2002;21:1094-9.
6. Whitson BA, Groth SS, Duval SJ, et al. Surgery for early-stage non-small cell lung cancer: a systematic review of the video-assisted thoracoscopic surgery versus thoracotomy approaches to lobectomy. Ann Thorac Surg 2008;86:2008-16.

7. Scott WJ, Allen MS, Darling G, et al. Video-assisted thoracic surgery versus open lobectomy for lung cancer: a secondary analysis of data from the American College of Surgeons Oncology Group Z0030 randomized clinical trial. J Thorac Cardiovasc Surg 2010;139:976-81.

8. Nwogu CE, D'Cunha J, Pang H, et al. VATS lobectomy has better perioperative outcomes than open lobectomy: CALGB 31001, an ancillary analysis of CALGB 140202 (Alliance). Ann Thorac Surg 2015;99:399-405.

9. Falcoz PE, Puyraveau M, Thomas PA, et al. Video-assisted thoracoscopic surgery versus open lobectomy for primary non-small-cell lung cancer: a propensity-matched analysis of outcome from the European Society of Thoracic Surgeon database. Eur J Cardiothorac Surg 2016;49:602-9.

10. Richardson VR, Cordell P, Standeven KF, et al. Substrates of Factor XIII-A: roles in thrombosis and wound healing. Clin Sci 2013;124:123-37.

11. Inbal A, Lubetsky A, Krapp T, et al. Impaired wound healing in factor XIII deficient mice. Thromb Haemost 2005;94:432-7.

12. Herouy Y, Hellstern MO, Vanscheidt W, et al. Factor XIII-mediated inhibition of fibrinolysis and venous leg ulcers. Lancet (London, England) 2000;355:1970-1.

13. Ogawa T, Morioka Y, Inoue T, et al. Involvement of blood coagulation factor XIII in burn healing in the carbon tetrachloride-induced hepatic injury model in rats. Inflamm Res 1995;44:264-8.

14. Cario E, Goebell H, Dignass AU. Factor XIII modulates intestinal epithelial wound healing in vitro. Scand J Gastroenterol 1999;34:485-90.

15. Saigusa S, Yamamura T, Tanaka K, et al. Efficacy of administration of coagulation factor XIII with definitive surgery for multiple intractable enterocutaneous fistulae in a patient with decreased factor XIII activity. BMJ Case Rep 2011. doi: 10.1136/bcr.09.2010.3342.

16. Fujita I, Kiyama T, Mizutani T, et al. Factor XIII Therapy of Anastomotic Leak, and Circulating Growth Factors. J Nippon Med Sch 2006;73:18-23.

17. Inoue $H$, Nishiyama N, Mizuguchi S, et al. Clinical value of exogenous factor XIII for prolonged air leak following pulmonary lobectomy: a case control study. BMC Surg 2014;14:109.

18. Clavien PA, Barkun J, de Oliveira ML, Vauthey JN, Dindo 
D, Schulick RD, et al. The Clavien-Dindo classification of surgical complications: five-year experience. Ann Surg 2009;250:187-96.

19. Rivera C, Bernard A, Falcoz PE, et al. Characterization and prediction of prolonged air leak after pulmonary resection: a nationwide study setting up the index of prolonged air leak. Ann Thorac Surg 2011;92:1062-8.

20. Fickenscher K, Aab A, Stüber W. A photometric assay for blood coagulation factor XIII. Thromb Haemost 1991;65:535-40.

21. Budczies J, Klauschen F, Sinn B V, et al. Cutoff Finder: a comprehensive and straightforward Web application enabling rapid biomarker cutoff optimization. van Diest $\mathrm{P}$, editor. PLoS One 2012;7:e51862.

22. Schroeder V, Kohler HP. Factor XIII: Structure and Function. Semin Thromb Hemost 2016;42:422-8.

23. Higaki S, Nakano K, Onaka S, et al. Clinical significance of measuring blood coagulation factor XIIIA regularly and continuously in patients with Crohn's disease. J Gastroenterol Hepatol 2006;21:1407-11.

24. D’Argenio G, Cosenza V, Riegler G, et al. Serum transglutaminase correlates with endoscopic and

Cite this article as: Shirai S, Yamauchi Y, Yokote F, Sakai T, Saito Y, Sakao Y, Kawamura M. Dynamics of coagulation factor XIII activity after video-assisted thoracoscopic lobectomy for non-small cell lung cancer. J Thorac Dis 2019;11(12):53825389. doi: $10.21037 /$ jtd.2019.12.08 histopathologic grading in patients with ulcerative colitis. Dig Dis Sci 2001;46:649-57.

25. Katona É, Nagy B, Kappelmayer J, et al. Factor XIII in bronchoalveolar lavage fluid from children with chronic bronchoalveolar inflammation. J Thromb Haemost 2005;3:1407-13.

26. Bagoly Z, Katona E, Muszbek L. Factor XIII and inflammatory cells. Thromb Res 2012;129 Suppl 2:S77-81.

27. Cordell PA, Kile BT, Standeven KF, et al. Association of coagulation factor XIII-A with Golgi proteins within monocyte-macrophages: implications for subcellular trafficking and secretion. Blood 2010;115:2674-81.

28. Kovar FM, Marsik CL, Jilma B, et al. The inflammatory response is influenced by FXIII VAL 34 LEU polymorphism in a human LPS model. Wien Klin Wochenschr 2009;121:515-9.

29. Ng CS, Whelan RL, Lacy AM, et al. Is minimal access surgery for cancer associated with immunologic benefits? World J Surg 2005;29:975-81.

30. Leaver HA, Craig SR, Yap PL, et al. Lymphocyte responses following open and minimally invasive thoracic surgery. Eur J Clin Invest 2000;30:230-8. 